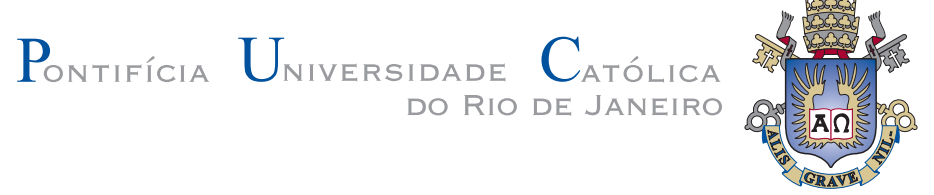

Thiago de Almeida Bastos

Campos de Distância Amostrados Adaptativamente com Aceleração por Placa Gráfica

Dissertação de Mestrado

Dissertação apresentada como requisito parcial para obtenção do grau de Mestre pelo Programa de Pós-graduação em Informática do Departamento de Informática da PUC-Rio

Orientador: Prof. Waldemar Celes Filho 


\title{
Campos de Distância Amostrados Adaptativamente com Aceleração por Placa Gráfica
}

Dissertação apresentada como requisito parcial para obtenção do grau de Mestre pelo Programa de Pós-graduação em Informática do Departamento de Informática do Centro Técnico Científico da PUC-Rio. Aprovada pela Comissão Examinadora abaixo assinada.

\author{
Prof. Waldemar Celes Filho \\ Orientador \\ Departamento de Informática — PUC-Rio \\ Prof. Marcelo Gattass \\ Departamento de Informática — PUC-Rio \\ Prof. Thomas Lewiner \\ Departamento de Matemática — PUC-Rio
}

Prof. Luiz Henrique de Figueiredo

IMPA

Prof. José Eugênio Leal

Coordenador Setorial do Centro Técnico Científico - PUC-Rio 
Todos os direitos reservados. É proibida a reprodução total ou parcial do trabalho sem autorização da universidade, do autor e do orientador.

\section{Thiago de Almeida Bastos}

Graduou-se em Bacharelado em Informática na PUC-Rio em 2005. Desde 2002 trabalha no laboratório de Computação Gráfica da universidade (Tecgraf) desenvolvendo sistemas de realidade virtual e visualização científica.

Ficha Catalográfica

Bastos, Thiago de Almeida

Campos de Distância Amostrados Adaptativamente com Aceleração por Placa Gráfica / Thiago de Almeida Bastos; orientador: Waldemar Celes Filho. — 2008.

v., 70 f.: il. (col.) ; $29,7 \mathrm{~cm}$

1. Dissertação (Mestrado em Informática) - Pontifícia Universidade Católica do Rio de Janeiro, Rio de Janeiro, 2008.

Inclui referências bibliográficas.

1. Informática - Teses. 2. Campos de distância amostrados adaptativamente. 3. Renderização de superfícies implícitas. 4. Programação em GPU. 5. Dispersão espacial perfeita. 6. Lançamento de raios em GPU. 7. Interpolação pseudotricúbica. I. Celes Filho, Waldemar. II. Pontifícia Universidade Católica do Rio de Janeiro. Departamento de Informática. III. Título. 
À minha mãe Maria João, meus avós Rita e João, e minha irmã Ana. 


\section{Agradecimentos}

À minha família e amigos, pelo grande apoio, sem o qual este trabalho não poderia ter sido realizado.

Ao meu orientador Waldemar Celes, pela grande dedicação, confiança e paciência; pelas excelentes idéias e pela orientação impecável.

Aos membros da banca e aos meus colegas, pelas várias sugestões e opiniões relevantes.

Ao Tecgraf e à PUC-Rio, pelos auxílios concedidos. 


\section{Resumo}

Bastos, Thiago de Almeida; Celes Filho, Waldemar. Campos de Distância Amostrados Adaptativamente com Aceleração por Placa Gráfica. Rio de Janeiro, 2008. 70p. Dissertação de Mestrado - Departamento de Informática, Pontifícia Universidade Católica do Rio de Janeiro.

A representação de formas é um problema fundamental em Computação Gráfica. Dentre as representações conhecidas para objetos tridimensionais, os campos de distância amostrados adaptativamente (ADFs) destacam-se por sua versatilidade. ADFs combinam os conceitos de geometria com dados volumétricos, permitem representar objetos com precisão arbitrária, e consolidam diversas operações - como visualização, modelagem de níveis de detalhe, detecção de colisão, testes de proximidade, metamorfose e operações booleanas - em uma única representação. Este trabalho propõe métodos para acelerar a reconstrução de ADFs estáticas, melhorar a qualidade dos campos reconstruídos, e visualizar iso-superfícies das ADFs, valendo-se do enorme poder computacional encontrado nas placas gráficas modernas (GPUs). Para que as ADFs sejam representadas de forma eficiente em placas gráficas, propõe-se o uso de uma estrutura hierárquica baseada em dispersão espacial perfeita. A renderização de ADFs é feita integralmente pela GPU, utilizando uma técnica de lançamento de raios baseada em traçado por esferas. Uma maneira de tratar as descontinuidades $C^{0}$ e $C^{1}$ inerentes às ADFs é sugerida para que o sombreamento das superfícies seja suave. Finalmente, o trabalho propõe um novo método de reconstrução para ADFs, capaz de representar melhor superfícies curvas. Os resultados são apresentados através de aplicações simples de visualização interativa, com ADFs geradas a partir de malhas de triângulos e sólidos primitivos.

\section{Palavras-chave}

Campos de distância amostrados adaptativamente. Renderização de superfícies implícitas. Programação em GPU. Dispersão espacial perfeita. Lançamento de raios em GPU. Interpolação pseudo-tricúbica. 


\section{Abstract}

Bastos, Thiago de Almeida; Celes Filho, Waldemar. GPUAccelerated Adaptively Sampled Distance Fields. Rio de Janeiro, 2008. 70p. MSc Thesis - Department of Computer Science, Pontifícia Universidade Católica do Rio de Janeiro.

Shape representation is a fundamental problem in Computer Graphics. Among known representations for three-dimensional objects, adaptively sampled distance fields (ADFs) are noted for their versatility. ADFs combine the concepts of geometry with volume data, allow objects to be represented with arbitrary precision, and consolidate several operations — such as visualization, level-of-detail modeling, collision detection, proximity tests, morphing and boolean operations - into a single representation. This work proposes methods to accelerate the reconstruction of static ADFs, to improve the quality of reconstructed fields, and to visualize ADF isosurfaces, making use of the massive computational power found in modern graphics hardware (GPUs). In order to efficiently represent ADFs on graphics cards, a hierarchical structure based on perfect spatial hashing is proposed. Rendering of ADFs is done completely on GPUs, using a ray casting technique based on sphere tracing. Means to overcome the $C^{0}$ and $C^{1}$ discontinuities inherent to ADFs are suggested in order to attain smoothly shaded iso-surfaces. Finally, a new reconstruction method for ADFs, which can better represent curved surfaces, is proposed. Results are presented through simple interactive visualization applications, with ADFs generated from both triangle meshes and primitive solids.

\section{Keywords}

Adaptively sampled distance fields. Implicit surface rendering. GPU programming. Perfect spatial hashing. GPU ray casting. Pseudo-tricubic interpolation. 


\section{Sumário}

1 Introdução 13

2 Trabalhos Relacionados $\quad 19$

2.1 Campos de Distância Tridimensionais $\quad 19$

$\begin{array}{lll}2.2 & \text { ADFs } & 21\end{array}$

2.3 Lançamento de Raios em GPU 28

3 Representação e Reconstrução de ADFs em GPU 29

$\begin{array}{lll}3.1 & \text { A Textura de Voxels } & 29\end{array}$

3.2 Visão Geral da Representação das ADFs 31

3.3 Octrees Baseadas em Dispersão Espacial 32

3.4 Dispersão Espacial Perfeita 34

3.5 Reconstrução do Campo de Distância 40

4 Visualização de ADFs em GPU 44

4.1 Traçado por Esferas $\quad 45$

4.2 Traçado por Esferas para ADFs Globais 46

4.3 Traçado por Esferas para ADFs de Fronteira 47

4.4 Descontinuidades 48

5 Resultados e Aplicações $\quad 51$

5.1 Construção de ADFs 51

5.2 Aplicações 53

6 Reconstrução Pseudo-Tricúbica $\quad 57$

$\begin{array}{ll}6.1 \text { Motivação } & 58\end{array}$

$\begin{array}{ll}6.2 \text { Implementação } & 59\end{array}$

$\begin{array}{lll}6.3 & \text { Resultados } & 61\end{array}$

7 Conclusão $\quad 63$

7.1 Trabalhos Futuros $\quad 64$

$\begin{array}{lc}\text { Referências Bibliográficas } & 65\end{array}$

A Código GLSL $\quad 69$ 


\section{Lista de figuras}

1.1 Objetos representados por superfícies poligonais e paramétricas. (Ambas as imagens são de domínio público e foram extraídas da Wikipédia.)

1.2 Sólidos representados por grades de voxels e CSG. (Imagens extraídas da Wikipédia.)

1.3 Visualização volumétrica de campos de distância 3-D. Imagens provenientes do trabalho [31].

2.1 Turbina representada por uma estrutura semelhante às ADFs. Imagens extraídas dos resultados do trabalho [3].

2.2 Letra "R" representada por uma ADF bidimensional. Imagens extraídas do trabalho [10].

2.3 Imagens renderizadas de ADFs, retiradas do trabalho [10].

2.4 Quatro níveis de detalhe extraídos da mesma ADF. Imagens retiradas do trabalho [10].

2.5 ADF de uma esfera com apenas dois níveis de subdivisão. 26

3.1 Uma quadtree (esquerda) e a sua textura de voxels (direita). 30

3.2 Visão geral da representação de ADF utilizada neste trabalho.

3.3 Ilustração em 2-D do acesso aos voxels nos vértices de uma célula de uma octree baseada em dispersão espacial.

3.4 Método de dispersão perfeita proposto por Lefebvre e Hoppe [20]. 36

4.1 Imagens renderizadas em GPU a partir de uma ADF de nível 8 do modelo "Eros". O modelo original é uma malha de triângulos e foi obtido do repositório Aim@Shape.

4.2 No traçado por esferas, os raios que "tangenciam" a superfície precisam de mais iterações para convergir.

4.3 Imagens coloridas de acordo com o número de iterações necessárias para que os raios interceptem as superfícies. As cores variam do verde claro (1 iteração) até o azul escuro (64 iterações).

4.4 Construção da textura de normais.

5.1 Estátua Tailandesa renderizada em GPU com normais suaves. $\quad 54$

5.2 Iso-superfícies do modelo Armadillo ("tatu") de Stanford. 55

5.3 Ramsés em quatro níveis de detalhes. 56

5.4 Interpolação entre ADFs, renderizada interativamente em GPU. $\quad 56$

6.1 Iso-superfície de um campo trilinear não-Euclidiano. Imagens divulgadas por Frisken et al. [10].

6.2 Célula de ADF, configurada com amostras do campo de distância de $\frac{1}{8}$ de uma esfera, plotada por três interpoladores diferentes.

6.3 Decomposição de uma interpolação 3-D em sub-interpolações unidimensionais. 
6.4 Comparativo entre ADFs trilineares (acima) e pseudo-tricúbicas (abaixo), utilizando a ADF de uma esfera em diferentes níveis de subdivisão. Neste exemplo, as ADFs trilineares foram renderizadas sem normais suaves.

6.5 Comparativo entre duas ADFs de nível 9. A ADF pseudo-tricúbica consegue representar mais detalhes, e a iluminação é naturalmente mais suave. 


\section{Lista de tabelas}

5.1 Resultados quantitativos para ADFs retratadas nesta dissertação. $\quad 53$

5.2 Desempenho da renderização de ADFs em GPU. 54

6.1 Comparativo de custos dos métodos de interpolação. 59

6.2 Comparativo entre o número de células das ADFs trilineares e pseudo-tricúbicas. Neste exemplo, todas as ADFs são superficiais. 61 
Clay is molded to form a cup, but it is on its non-being that the utility of the cup depends. Doors and windows are cut out to make a room, but it is on its non-being that the utility of the room depends. Therefore turn being into advantage, and turn non-being into utility.

Lao Tzu, Tao Te Ching, Ch. 11. 